\title{
Quadtree Decomposition Texture Analysis in Paper Formation Determination
}

\author{
Erik Lieng \\ Norwegian University of Science and Technology \\ Department of Engineering Cybernetics, NO-7491 Trondheim, Norway \\ Erik.Lieng@itk.ntnu.no
}

\begin{abstract}
The main topic of the article is to give a detailed description of the new and promising quadtree decomposition texture analysis method used for paper formation determination. Paper formation or configuration of fibers, fines and fillers in the two-dimensional spatial xy-domain of the paper is a very important property and image analysis application for the paper industry. The basis of the method is the successive quadtree decomposition process resulting in a two-dimensional block partitioning of the formation structure image analysed. In this context the blocks represents a unit of variation, and the size of a quadtree block is controlled by a set of different parameters. In addition to the primary features detected by the algorithm, characterization of a large set of secondary features is performed, including gradient analysis and spatial distribution analysis.
\end{abstract}

\section{Introduction}

The work is a part of a larger project at PFI with the objective to find and evaluate new ways to determine formation and performing formation analysis. Matlab has been used as a development tool and several graphical user interfaces (GUIs) has been created. The methods developed are also valuable for analysing e.g. print quality assessments in complex images, a work that will be published later.

Normally the formation property is measured by the standard deviation value of the evaluated image or a closely related parameter [1-4], a simple and straight forward method which do not give information about size features in the image or spatial distributions in the two-dimensional spatial domain. The quadtree method in contradistinction produces information about size features and spatial distributions and an overview of the possibilities of the software routines developed can be described in the following list of features:

1. Block size class measurements

2. Distribution statistics

a. Spreading of blocks within the image frame

b. Locating clusterings or groupings of the blocks

c. Nearest-neighbour measurements to locate patterns in the formation

3. Discrimination between good and poor formation structure 
4. Gradient analysis

The algorithm for decomposition can be descirbed with the following properties:

1. Recursive successive algorithm

2. Rectangular block partitioning

3. Quadtree structure

4. Recomposition

The quadtree decomposition algorithm have been reported and applied in a wide range of signal processing applications [5], texture analysis [6], image processing $[7,8]$ and data mining and data structure $[9,10]$, and is an intuitive approach for spatial decomposition and an analytical tool that can be applied to detect different types of two-dimensional (2D) features in the paper structure.

\section{Methods and Experimental}

\subsection{Acquisition of Formation Images}

The image acquisition was done in two steps. First the beta formation images collected and secondly the optical images were acquired. The acquisition of the beta formation images was done using the Fujifilm BAS-1800/FORMEX system. The system uses Imaging Plates (IP) to record the beta ray signal in the Fujifilm FORMEX unit. The Imaging Plates were then scanned in the Fuji BAS-1800 scanner. The pixel resolution was $50 \mu \mathrm{m}$. The image intensities were corrected according to the simultaneously recorded Mylar film weights with grammages equivalent to 50, 100,150 and $200 \mathrm{~g} / \mathrm{m}^{2}$. The acquisition of the optical images was done by using the high-resolution digital Eyelike camera. The spatial resolution was $50 \mu \mathrm{m}$. Background correction was used to eliminate for non-uniformities in the light source. The optical intensity levels of the individual samples agreed well with calibrated optical measurements done on the Tapio Formation analyser.

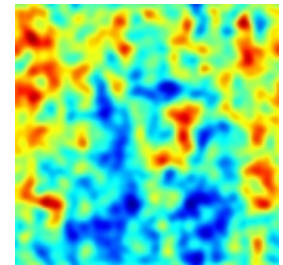

a)

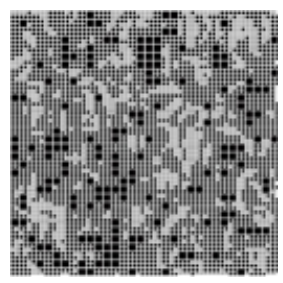

b)

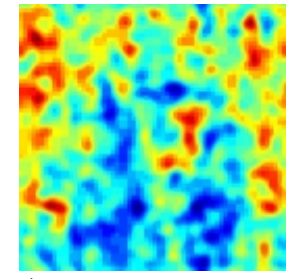

c)

Fig. 1. a) Formation input image. b) Quadtree block decomposition image. c) Recomposited formation image. 


\subsection{The Quadtree Decomposition Algorithm}

The quadtree decomposition are performed by a successively refinement algorithm. First the complete formation texture image is evaluated. The difference between maximum and minimum, and the preset threshold value are compared. If the difference exceeds, the image frame is divided into four equal subimages. The new subimages is then evaluated separately, and the consecutive process stops then the difference is not exceeded or there are one single pixel left.

The quadtree structure from the starting point to the end forms a quadtree datastructure of subimages. If $\mathrm{d}$ is the number of dimensions, each node in the tree can be splitted into $2^{\mathrm{d}}$ children. For two-dimensional images the $\mathrm{d}$ parameter is 2 .

\subsection{Additional Criteria of Decomposition}

Spatial criteria for decomposition using the dynamic range as an input variable to the algorithm:

- Maximum greylevel difference (maximum - miniumum) within the produced subimages

- Standard deviation cutoff

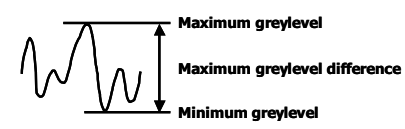

Fig. 2. Maximum and minimum properties used as a criterion for the quadtree decomposition algorithm.

Frequency criteria for decomposition:

- Frequency bandwidth cutoff

- Spectral density cutoff

- Subband frequency cutoff

The resulting decomposition subimages of different blocksizes consists of features of equal or similar properties, that characterizes the paper structure sample variation distributed in the two dimensional spatial domain. 


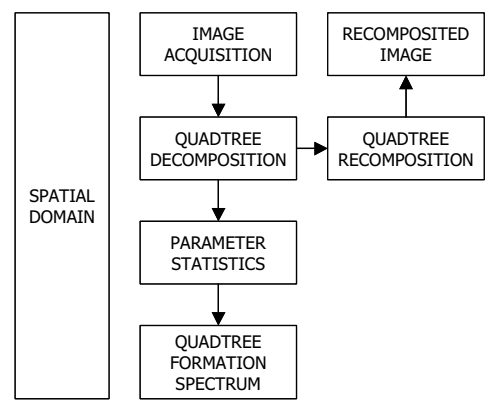

Fig. 3. Overview flow chart of the analysis.

\section{Results and Discussion}

\subsection{Output Parameters and Statistics}

Standard features and output parameters from the formation analysis algorithms includes the following list of features and parameters:

1. Quadtree formation spectrum

2. Number of equivalent blocks for each size class

3. Total number of blocks in decomposition

4. Total area of each equivalent block size class

5. Centre of gravity for each block size class

6. Spreading factor for each block size class

7. Number of four and eight way connected equivalent blocks

\subsection{Optimal Discrimination between Samples}

An important feature in the formation analysis is the analysators ability to discriminate optimally between samples in the sample dataset. Figure 4 shows a discrimination test performed on a large set of commercial newsprint samples. 


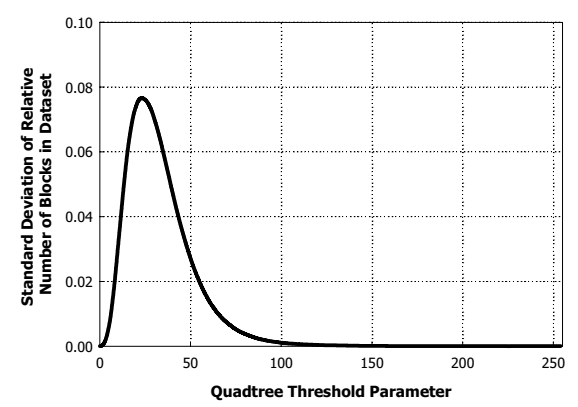

Fig. 4. Discrimination test verification plot for the dataset.

The discrimination test shows that it is possible, depending on the selection of input parameters that controls the decomposition process, to obtain an optimal separation between the samples in the dataset.

The relation to standard formation measurement parameters has been verified. The conclusion is that the quadtree decomposition have the full capability to perform a distinct analysis of the partially random structure of formation as showed in figure 5, were the derived quadtree threshold parameters are calculated from the relative number of blocks $50 \%$-value.

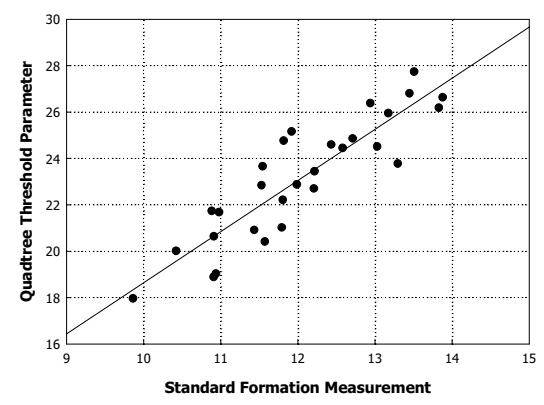

Fig. 5. The relation between quadtree analysis and standard formation measurement. The quadtree threshold parameter derived from the relative number of blocks $50 \%$-value. Statistical correlation coefficient between the datasets is RSQ $=0.80$.

The linear regression plotted in figure 5 is analytically given by the equation $Y=2.2045 X-3.4036$, were $X$ represent the standard formation measurement data and $\mathrm{Y}$ the derived quadtree threshold parameter data, resulting in a RSQ of 0.80 . In figure 6 the outermost points in the plot in figure 5 are selected representing the lowest and highest standard formation values. 


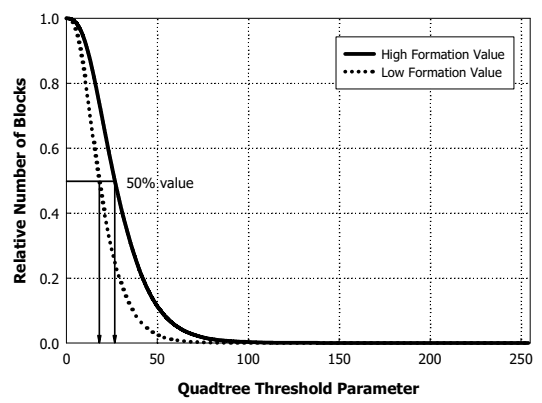

Fig. 6. The principal method for determination the 50\%-values from the relative number of blocks for the respective lowest and highest formation values.

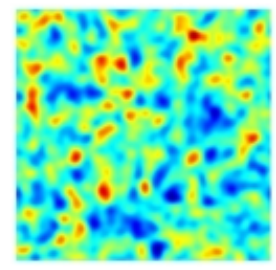

a)

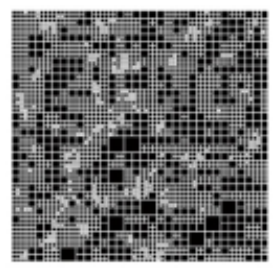

b)

Fig. 7. a) Good paper formation ranked image. b) Respective quadtree decomposition block structure.

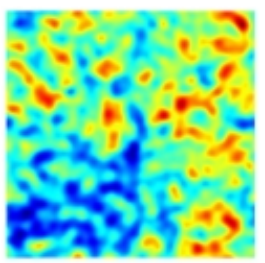

a)

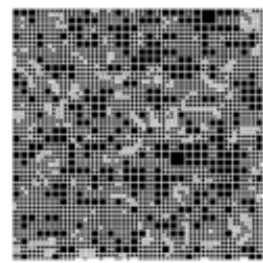

b)

Fig. 8. a) Poor paper formation ranked image. b) Respective quadtree decomposition block structure.

The lowest standard formation value represents the best paper formation, hereafter denoted good formation, and equivalently the highest standard formation value represents the poorest paper formation, denoted poor formation. In figure 7 and 8 respectively examples of good and poor formation and their respective quadtree decompositions are given. From the quadtree decomposition the quadtree formation spectrum is derived. The quadtree formation spectrum constitutes of blocksize distribution curves as illustrated in figure 9 a) and b) respectively. 

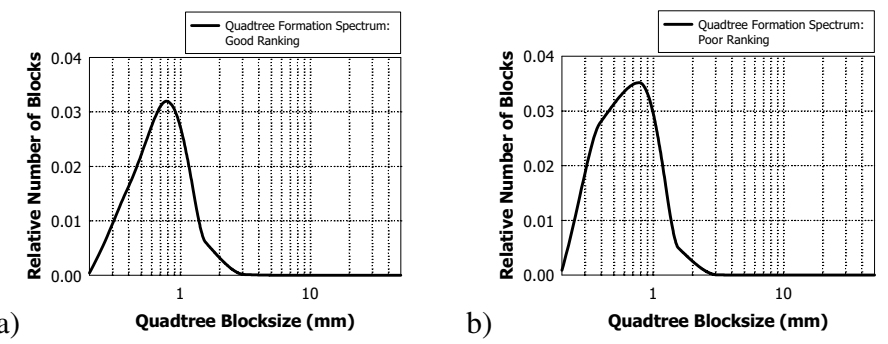

Fig. 9. a) Blocksize distribution from the good paper formation image decomposition. b) Blocksize distribution from the poor paper formation image decomposition.

Good formation shows a blocksize distribution with weight on large blocksizes and a distribution shifted towards the right and reduced amount of small blocksizes, implying more homogenic formation. Poor formation shows a blocksize distribution with weight on smaller blocksizes and a distribution shifted towards the left and a higher amount of small blocksizes, implying more inhomogenic formation.

\subsection{Gradient Features with Focus on Floc Hardness and Softness Measurements}

A secondary effect of the quadtree decomposition is the gradient features it provides as showed in figure 10. The floc edges have different degrees of decomposition depending on the hardness or softness of the floc edge.

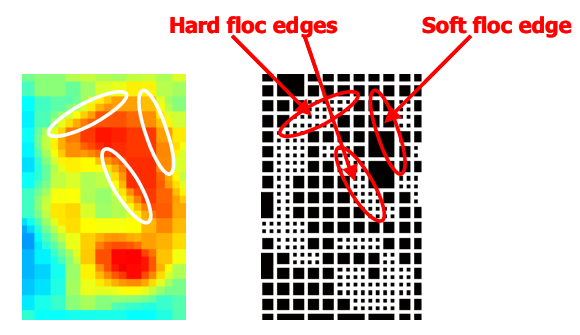

Fig. 10. Floc edges of different gradients will show different degree of decomposition. A hard floc edge will show a high degree of spatial decomposition. A soft floc edge will show a low degree of decomposition.

\subsection{Brightness and Contrast Dependency}

The results also show that the decomposition is brightness independent, meaning that the average greylevel in the input image do not alter the decomposition results. The decomposition is however contrast dependent, meaning that a change in contrast for the input images do alter the resulting decomposition output. A calibration routine in the image acquisition process is therefore recommended. 
Although the examples and the theory in this article are derived from commerical newsprint paper grade samples, the analysis is applicable to other paper types as well.

\section{Conclusions and Final Remarks}

The conclusion is that the quadtree decomposition texture analysis is a useful and valuable tool in analysis of the paper structure.

The quadtree decomposition is a recursive successive decomposition algorithm that performs a rectangular block partition of the formation (sub)images. The subimages have a quadtree datastrucutre which can be recomposed by an inverse algorithm.

A large number of output parameters may be derived from the quadtree paper formation analysis and includes:

- Quadtree formation spectrum

- Number of equivalent blocks for each size class

- Total number of blocks in decomposition

- Total area of each equivalent block size class

- Centre of gravity for each block size class

- Spreading factor for each block size class

- Number of four and eight way connected equivalent blocks

In addition to these parameters the quadtree paper formation analysis performs discrimination between rankings of formation textures and gradient analysis of floc edge properties.

\section{Further Work}

Further implementation of the quadtree decomposition methods may be done offline or online in the paper mill for paper formation analysis and quality purposes, to analyse paper specific parameters not possible by other methods available in formation analysis. The quadtree decomposition analysis method provides a local and real twodimensional (2D) analysis of the paper structure.

\section{References}

1. Kajanto, I.M., Komppa, A., Ritala, R.K., "How formation should be measured and characterized”, Nord. Pulp Pap. Res. J., 4(3), pp 219-228, (1989)

2. Gullichsen, J., Paulapuro, H., Niskanen, K., "Papermaking science and technology 16. Paper physics", Fapet Oy, Helsinki, Finland, 324pp, (1998)

3. Johansson, P., Norman, B., "Methods for evaluating formation, print unevenness and gloss variation developed at STFI", 1996 Process and product quality conference, Cincinnati, OH, USA, pp 139-145, 14-17 Oct., (1996) 
4. Kajanto, I., Komppa, A., Ritala, R.K., "The application determines how formation should be measured and characterized", Paper presented at XXIII EUCEPA Conference, Harrogate, UK, vol. 2, pp 624-636, 31 May-3 June, (1988)

5. Gibson, J.D., Berger, T., Lookabaugh, T., Lindbergh, D., Baker, R.L., "Digital Compression for Multimedia Principles and Standards", Morgan Kaufmann Publishers, (1998)

6. Ofek, E., Shilat, E., Rappoport, A., Werman, M., "Highlight and Reflection-Independent Multiresolution Textures from Image Sequences", IEEE Computer Graphics and Applications, vol.17 (2), March-April, (1997)

7. Rehrauer, H., Seidel, K., Datcu, M., "Multiscale Image Segmentation with a Dynamic Label Tree", IGARSS'98, (1998)

8. Smith, J., "Integrated Spatial and Feature Image Systems: Retrieval, Analysis and Compression," Ph.D. Dissertation, Columbia University, (1997)

9. Watt, A., "3D Computer Graphics", 3rd ed., Addison-Wesley, (2000)

10. Keim, D., Hinneburg, A., "Clustering techniques for large data sets: From the past to the future", SIGKDD 1999 International Conference on Knowledge Discovery and Data Mining, San Diego, California, USA, (1999) 